\title{
Síndrome da visão do computador: fatores de risco associados e intervenções ergonômicas efetivas para sua manutenção
}

\author{
Computer vision syndrome: associated risk factors and effective ergonomic interventions \\ for its maintenance
}

\section{Síndrome de visión artificial: factores de riesgo asociados e intervenciones ergonómicas efectivas para su mantenimiento}

Indiara Iris de Oliveira Araújo ${ }^{1 *}$, Jemima Ferreira Coelho¹, Livia Oliveira Delgado Mota.

\section{RESUMO}

Objetivo: Analisar os fatores de risco relacionados a Síndrome da Visão do Computador (SVC), bem como quais intervenções ergonômicas são efetivas na manutenção dessa patologia e melhora da qualidade de vida. Métodos: Trata-se de uma revisão integrativa da literatura que levantou dados bibliográficos sobre os fatores de risco e intervenções ergonômicas da SVC. Os resultados da revisão revelaram 21 publicações, no período de 2010 a 2020, que apresentaram adequação com os critérios de inclusão estabelecidos. Resultados: São vários os fatores de risco relacionados a SVC. Fatores ambientais: aqueles relacionados ao uso do computador, relacionados com hábitos de visualização e ao local de trabalho. Fatores individuais: maior prevalência de sintomas da SVC no gênero feminino, além de problemas refrativos, olhos secos, e fatores musculo esqueléticos. A melhor abordagem à SVC inclui intervenções ao nível da ergonomia do posto de trabalho, da correção refrativa, da educação do utilizador, da iluminação e do planeamento das pausas durante as tarefas de visualização ao computador. Considerações finais: Portanto, sendo um resultado de multi causas, a SVC exige uma intervenção sob medida para uma prevenção e tratamento eficazes, melhorando a qualidade de vida dessa população.

Palavras-chave: Síndrome visão computador, Ergonomia, Saúde trabalhador.

\begin{abstract}
Objective: To analyze the risk factors related to Computer Vision Syndrome (CVS), as well as which ergonomic interventions are effective in maintaining this pathology and improving quality of life. Methods: This is an integrative literature review that collected bibliographic data on risk factors and ergonomic interventions in CVS. The results of the review revealed 21 publications, from 2010 to 2020 , which were adequate with the established inclusion criteria. Results: There are several risk factors related to CVS. Environmental factors: those related to computer use, related to viewing habits and the workplace. Individual factors: higher prevalence of CVS symptoms in females, in addition to refractive problems, dry eyes, and musculoskeletal factors. The best approach to CVS includes interventions in terms of workplace ergonomics, refractive correction, user education, lighting and planning of breaks during computer visualization tasks. Final considerations: Therefore, being a result of multiple causes, SVC requires a tailored intervention for effective prevention and treatment, improving the quality of life of this population.
\end{abstract}

Key words: Computer vision syndrome, Ergonomics, Worker's health.

1 Universidade de Vassouras, Vassouras - RJ.
${ }^{*}$ E-mail: indiaraaraujo28@gmail.com

SUBMETIDO EM: 8/2021

ACEITO EM: 9/2021

PUBLICADO EM: 10/2021 


\section{RESUMEN}

Objetivo: Analizar los factores de riesgo relacionados con el Síndrome de Visión por Computador (SVC), así como qué intervenciones ergonómicas son efectivas para mantener esta patología y mejorar la calidad de vida. Métodos: Se trata de una revisión integradora de la literatura que recopiló datos bibliográficos sobre factores de riesgo e intervenciones ergonómicas en la SVC. Los resultados de la revisión revelaron 21 publicaciones, de 2010 a 2020, las cuales fueron adecuadas a los criterios de inclusión establecidos. Resultados: Existen varios factores de riesgo relacionados con la SVC. Factores ambientales: los relacionados con el uso de la computadora, relacionados con los hábitos de visualización y el lugar de trabajo. Factores individuales: mayor prevalencia de síntomas de SVC en mujeres, además de problemas refractivos, ojos secos y factores musculoesqueléticos. El mejor enfoque de SVC incluye intervenciones en términos de ergonomía en el lugar de trabajo, corrección refractiva, educación del usuario, iluminación y planificación de descansos durante las tareas de visualización por computadora. Consideraciones finales: Por tanto, al ser el resultado de múltiples causas, la SVC requiere de una intervención a medida para una prevención y tratamiento eficaces, mejorando la calidad de vida de esta población.

Palabras clave: Síndrome de visión por computador, Ergonomía, Salud del trabajador.

\section{INTRODUÇÃO}

O mundo moderno conta com a evolução da tecnologia para agilizar e desempenhar as tarefas profissionais, assim como com a comodidade que os equipamentos eletroeletrônicos trazem aos processos de trabalho. Neste contexto, porém, a sabotagem com a saúde em prol da sobrevivência no mercado de trabalho, determina sérias consequências à qualidade de vida do trabalhador. $O$ uso do computador e telas exige que o ser humano se submeta diariamente a períodos prolongados diante do equipamento (NEVES LRC, et al., 2019; MUNSHI S, et al., 2017).

Diante do uso prolongado do computador, surgiu a síndrome da visão do computador (SVC), caracterizada por sintomas visuais, oculares, que incluem a fadiga visual, irritação ocular, prurido, olhos secos, sensação de ardor, vermelhidão, lacrimejamento, fotofobia, dor no pescoço e costas, entre outros (NEVES LRC, et al., 2019; RANDOLPH AS, 2017).

Além disso fatores ambientais, como o clima, correntes de ar e iluminação, fatores fisiológicos ou físicos, como posição da tela do computador, posições ergonômicas da pessoa, brilho da tela do computador, e ainda fatores biológicos, como problemas refrativos, olhos secos e problemas ortopédicos contribuem para a aparição da SVC (SÁ EC, et al., 2012; GOWRISANKARAN S e SHEEDY JE, 2015)

Atualmente existe uma estimativa de que $90 \%$ das pessoas que utilizam o computador por um período maior do que 3 horas diárias apresenta algum sintoma da SVC. Assim essa síndrome vem se tornando um problema de saúde pública em todo o mundo, porém a grande desinformação sobre está síndrome dificulta seu diagnóstico e tratamento, afetando a qualidade de vida (ROSIENFIELD M, 2016).

O presente estudo possui o objetivo de analisar os fatores de risco relacionados a essa SVC, bem como quais intervenções ergonômicas são efetivas na manutenção dessa patologia e melhora da qualidade de vida.

\section{MÉTODOS}

Este estudo trata-se de uma revisão integrativa da literatura que objetivou analisar os fatores de risco e intervenções ergonômicas da Síndrome da visão do Computador em estudos desenvolvidos no Brasil e em outros países do mundo. Para tanto foi necessário: realizar levantamento do estudo; sistematizar a metodologia para o desenvolvimento da revisão integrativa; buscar as publicações nas bases de trabalhos acadêmicos; classificar as publicações; fazer a leitura e análise crítica dos estudos incluídos. 
Os resultados da revisão integrativa da literatura revelaram 21 publicações que apresentaram adequação com os critérios de inclusão estabelecidos, bem como com a questão norteadora. A análise realizada pode auxiliar pesquisadores que tem interesse em pesquisa da SVC realizadas no Brasil e em outros países do mundo, sem precisar realizar todo o procedimento de revisão da literatura, uma vez que isso já foi feito e está apresentado neste estudo.

Foram buscados trabalhos publicados no período de 2010 a 2020 nas bases de dados Google Acadêmico, LILACS, Pubmed. Na base de dados Google Acadêmico com as palavras chaves: "síndrome visão computador, ergonomia, saúde trabalhador" foram encontrados 10 resultados. No LILACS foi buscado por "computer vision syndrome" sendo encontrados 27 artigos publicados na íntegra entre 2010 a 2021; já no Pubmed com a palavra-chave "computer vision syndrome" foram encontrados 115 artigos no período de tempo estipulado (Figura 1). Foram incluídos artigos que abordaram os sintomas da síndrome da visão do computador; os fatores de risco da síndrome visual do computador e as intervenções ergonômicas para a SVC.

Como critério de exclusão foram eliminados os artigos que tangenciam a temática, que abordam a síndrome visual não relacionada ao computador, publicações sem relação significativa com as palavras-chave (considerar sinônimos), publicações duplicadas.

Figura 1 - Fluxograma de seleção de artigos.
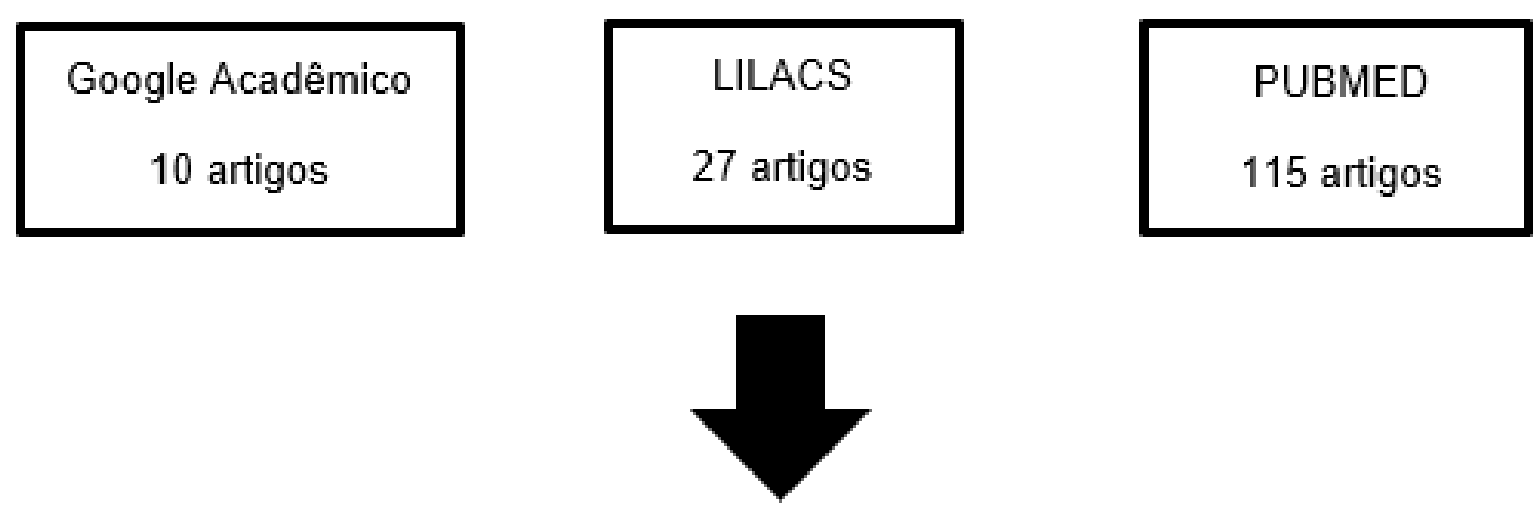

Critérios de inclusão: Foram incluídos artigos que abordaram os sintomas da síndrome da visão do computador; os fatores de risco da síndrome visual do computador e as intervenções ergonômicas para a SVC

Critérios de exclusão: foram eliminados os artigos que tangenciam a temática, que abordam a síndrome visual não relacionada ao computador, publicações sem relação significativa com as palavras-chave (considerar sinônimos), publicações duplicadas.

Obteve-se um total de 21 artigos, após análise dos critérios.

Fonte: Araújo IIO, et al., 2021.

\section{RESULTADOS E DISCUSSÃO}

Em 2007, os sintomas da SVC ocorriam em cerca de 75 a $90 \%$ dos usuários de computador e telas, e cerca de $22 \%$ apresentavam sintomas musculoesqueléticos. Contudo em 2016, constatou-se que cerca de $65 \%$ dos indivíduos em uma pesquisa que envolvia 10.000 habitantes, apresentavam sintomas da SVC (LURATI AR, 2018). 
São vários os fatores de risco relacionados a SVC, sendo agrupados da seguinte forma: fatores de risco individuais e fatores de risco ambientais. Sendo os fatores ambientais: aqueles relacionados ao uso do computador e telas, relacionados com hábitos de visualização e os relacionados ao local de trabalho. Quanto aos fatores de risco individuais, alguns estudos sugerem maior prevalência de sintomas da SVC no gênero feminino em relação ao gênero masculino devido as alterações hormonais, as quais afetam a produção de lagrimas levando a sintomas como olho seco. Este fato pode estar relacionado a prevalência elevada de olho seco entre os sujeitos do género feminino (ROSENFIELD M, 2011; RANASINGHE P, et al., 2016; PORTELLO JK, et al., 2012).

Além do gênero, o aumento da idade do usuário em uso do computador parece ter relação com o olho seco, levando a SVC, assim foi criado o índice de doença da superfície ocular (IDSO), uma escala a qual afere o olho seco, verificando o aumento deste na população feminina. Também deve ser considerado o efeito do envelhecimento do sistema visual. Existe ainda uma correlação estatisticamente significativa entre a idade e o IDSO. Não menos importante constatou-se que a prevalência da SVC em pessoas com patologias oculares pré-existentes, mais comumente miopia e presbiopia, é maior do que em pessoas sem doença ocular (ROSENFIELD M, 2011; RANASINGHE P, et al., 2016; PORTELLO JK, et al., 2012).

O esforço acomodativo entre o presbiope e os usuários de tela aumentam. A presbiopia foi identificada como um importante fator associado à alta incidência de astenopia. Isto é, mesmo uma pequena quantidade de erro refratário de igual a ou mais de $0,5 \mathrm{D}$ de miopia, hipermetropia ou astigmatismo aumentam o desconforto subjetivo com o uso de computadores, evidências de erros astigmáticos de até $0,12 \mathrm{D}$ aumentam a fadiga ocular, estabelecendo o fato de que mesmo uma quantidade mínima de ametropia é um fator que tem um impacto negativo no conforto visual. Erros de refração corrigidos de forma adequada, com um aumento significativo na amplitude, velocidade e tempo de acomodação e relaxamento explica a redução significativa de sintomas astenópicos subjetivos com a intervenção acima. Uma maior satisfação entre os usuários de computadores e similares ainda pode ser alcançada através de lentes presbiópicas cobrindo um alcance de visão de até $1 \mathrm{~m}$ (PARILHAR JKS, et al., 2016).

O fator que mais contribui para os sintomas da síndrome da visão do computador parece ser o olho seco, que resulta em desconforto, distúrbios visuais e instabilidade do filme lacrimal. Alguns fatores contribuem para que ocorra um desequilíbrio desse processo, podendo se destacar a baixa frequência do piscar. Em média, um ser humano pica 15 vezes por minuto, alguns estudos indicam que a taxa de intermitência no computador é menor do que o normal. Em conjunto a isso, os usuários de computador geralmente veem seu material de leitura com um olhar horizontal, resultando em um aumento da fenda palpebral e da área da superfície ocular exposta aos efeitos do ambiente, contribuindo para uma má qualidade do filme lacrimal e estresse temporário da córnea, resultando nos sintomas de olho seco (GENTIL RM, et al., 2011; SANCHEZ V, et al., 2020).

Alguns estudos sugerem que mulheres em período da menopausa têm maior probabilidade de desenvolver este quadro, porém não há ainda uma explicação fisiopatológica para o fato. De maneira semelhante, também existem estudos que identificam alguns medicamentos que aumentam o risco de lesão ocular, e sucessivamente na visão (como diuréticos, anti-histamínicos, antidepressivos e antipsicóticos) mas, sem uma explicação etiológica. Outros ainda destacam o tabagismo ou patologias como, Diabetes Mellitus, artrite, SIDA, doenças oculares já presentes anteriormente, no sentido de poderem agravar a sintomatologia, mas também não há registro em concreto do mecanismo pelo qual tal fato acontece (CRUZ C, et al 2018).

Nos últimos anos, o uso de aparelhos eletrônicos portáteis aumentou. O uso desses dispositivos apresenta desafios visuais diferentes em comparação com uma tela fixa. Por exemplo, display eletrônico portátil, como leitores eletrônicos, pode ser usado sob uma luz com condições de funcionamento diferente e podem estar associados a maiores riscos de desconforto devido à iluminação inadequada (GOWRISANKARAN $S$ e SHEEDY JE, 2015).

Além disso, ao contrário dos tradicionais computadores, dispositivos portáteis podem ser usados em diferentes distâncias e, muitas vezes, requerem tamanhos de fonte menores para o tamanho da tela, o que pode impor variações nas demandas do sistema visual. Um estudo recente determinou a distância de visualização preferida para smartphones em 129 adultos visualmente normais e descobriram que a distância 
de visualização dependia da natureza da tarefa e o tamanho do texto. O tamanho médio da fonte preferida e a distância do smartphone quando comparados aos computadores são parecidas, entretanto ainda existem poucos estudos analisando os sintomas da SVC em uso de aparelhos portáteis por longos períodos de tempo (GOWRISANKARAN S e SHEEDY JE, 2015).

Outros fatores, como fatores ergonômicos, estão relacionados ao desconforto e à fadiga visual. A iluminação é uma das causas que influencia significativamente nos danos aos olhos. Uma distribuição errada de iluminação no campo visual pode causar brilho excessivo e levar a fadiga ocular. $O$ filtro antirreflexo não reduz os sintomas de astenopia, mas foi constatado que diminui o brilho e aperfeiçoa o contraste da tela, afirmando-se como um meio eficaz para eliminar reflexos, melhorando o conforto visual. Já em relação ao posicionamento da tela, pesquisas sugerem que as distâncias de $35-40 \mathrm{~cm}$ entre os olhos do indivíduo e a tela resultam em menor número de queixas e diminui o esforço visual. Como também, a tela posicionada em lugares mais altos tem maior relação com o cansaço visual (GENTIL RM, et al., 2011; AL RASHIDI SH e ALHUMAIDAN H, 2017).

Um estudo transversal envolvendo 713 universitários na Arábia Saudita, constou que o sintoma mais comum devido ao uso prolongado do computador foi dor no pescoço ou no ombro, relatado por $82,2 \%$. No geral, $66,5 \%$ dos indivíduos sofriam de dor de cabeça e 51,5\% de olhos secos, em leve, moderada ou grave formato. O uso de dispositivos eletrônicos por mais de $5 \mathrm{~h}$ também foi associado a sintomas de SVC (MOWATT L, et al., 2017).

Outro estudo com 409 alunos da Faculdade de Ciências Médicas da Universidade de Índias Ocidentais (UWI), Jamaica. Constou que dor no pescoço, cansaço visual, dor no ombro e olhos queimando foram os sintomas de SVC mais comuns. Olhos secos, visão dupla e visão turva foram os sintomas menos comumente experimentados, sendo mais prevalentes naqueles que seguraram a tela em angulação não recomendada. Em ambos os estudos a prevenção da síndrome da visão computacional se daria por práticas ergonômicas adequadas diminuindo assim a maioria dos sintomas apresentados pelos estudantes (TAWIL LA, et al., 2018). 
Quadro 1 - Principais resultados encontrados na literatura.

\begin{tabular}{|c|c|c|c|c|}
\hline $\begin{array}{l}\text { Autores e Ano de } \\
\text { Publicacão }\end{array}$ & Titulo & Tipo de estudo & Resultados & Conclusão \\
\hline $\begin{array}{l}\text { PORTELLO JK, et al., } \\
2012\end{array}$ & $\begin{array}{l}\text { Computer-related } \\
\text { visual symptoms in } \\
\text { office workers }\end{array}$ & $\begin{array}{l}\text { Um estudo } \\
\text { quantitativo } \\
\text { transversal aplicado } \\
\text { em funcionários de } \\
\text { escritórios da cidade } \\
\text { de Nova York. }\end{array}$ & $\begin{array}{l}\text { Foi observada uma correlação positiva entre sintomas e } \\
\text { a quantidade de horas gastas trabalhando em um } \\
\text { computador em um dia normal. O sintoma mais } \\
\text { prevalente foi olhos cansados, seguido por olho seco e } \\
\text { desconforto ocular, respectivamente. Sintomas } \\
\text { variaram com o gênero, sendo maior nas mulheres, } \\
\text { etnia, sendo maior em hispânicos. }\end{array}$ & $\begin{array}{l}\text { Os sintomas visuais ocorrem com frequência } \\
\text { na população em geral produzindo desconforto } \\
\text { por longos períodos de tempo. Eles estão } \\
\text { fortemente associados a doenças da superfície } \\
\text { ocular. Novas ações terapêuticas precisam ser } \\
\text { desenvolvidas para essa condição. }\end{array}$ \\
\hline $\begin{array}{l}\text { PARILHAR JKS, et } \\
\text { al., } 2016\end{array}$ & $\begin{array}{l}\text { Computer \& visual } \\
\text { display terminals } \\
\text { (VDT) vision } \\
\text { syndrome (CVDTS) }\end{array}$ & $\begin{array}{l}\text { Este artigo trata-se } \\
\text { de uma revisão de } \\
\text { literatura a qual tem o } \\
\text { foco nos vários } \\
\text { aspectos da tela do } \\
\text { computador assim } \\
\text { como na síndrome } \\
\text { dos terminais descrita } \\
\text { na literatura anterior. }\end{array}$ & $\begin{array}{l}\text { Esta síndrome está ganhando importância devido ao } \\
\text { uso generalizado de tecnologias no dia a dia. Está } \\
\text { associada a sintomas astenópicos, embaçamento } \\
\text { visual, olhos secos, musculoesquelético, sintomas como } \\
\text { dor no pescoço, dor nas costas, dor no ombro, síndrome } \\
\text { do túnel do carpo, psicossomose fatores sociais, } \\
\text { tromboembolismo venoso, tendinite do ombro e } \\
\text { epicondilite do cotovelo. Apropriado identificação de } \\
\text { sintomas e fatores causais são necessários para o } \\
\text { diagnóstico preciso e gestão. }\end{array}$ & $\begin{array}{l}\text { Auxiliares de refração, uso de monitores de } \\
\text { LED, filtros de tela, melhorando a iluminação } \\
\text { do ambiente, contribuem para melhora dos } \\
\text { fatores musculo esqueléticos. Uso de } \\
\text { colírios lubrificantes, tratamento de qualquer } \\
\text { outro distúrbio da superfície ocular trazem } \\
\text { benefícios na manutenção da superfície } \\
\text { ocular. }\end{array}$ \\
\hline $\begin{array}{l}\text { MUNSHI S, et al., } \\
2017\end{array}$ & $\begin{array}{l}\text { Computer vision } \\
\text { syndrome - A } \\
\text { common cause of } \\
\text { unexplained visual } \\
\text { symptoms in the } \\
\text { modern era } \\
\end{array}$ & $\begin{array}{l}\text { As informações foram } \\
\text { coletadas no Medline, } \\
\text { Embase \& National } \\
\text { Library of Medicina } \\
\text { nos últimos } 30 \text { anos } \\
\text { até março de } 2016 . \\
\end{array}$ & $\begin{array}{l}\text { A SVC envolve várias especialidades. Embora seja uma } \\
\text { condição comum, há pouca consciência entre a } \\
\text { população e os profissionais da saúde. É importante } \\
\text { identificar corretamente a diferença entre a SVC dos } \\
\text { ataques isquêmicos transitórios e amaurose fugax, } \\
\text { evitando terapias desnecessárias. }\end{array}$ & $\begin{array}{l}\text { A maior conscientização da população sobre } \\
\text { a SVC e a educação de profissionais de saúde } \\
\text { é vital. As estratégias preventivas devem } \\
\text { compreender desde a parte ergonômica do } \\
\text { local de trabalho. }\end{array}$ \\
\hline $\begin{array}{l}\text { MOWATT L, et al., } \\
2017\end{array}$ & $\begin{array}{l}\text { Computer vision } \\
\text { syndrome and } \\
\text { ergonomic practices } \\
\text { among } \\
\text { undergraduate } \\
\text { university students }\end{array}$ & $\begin{array}{l}\text { Estudo transversal } \\
\text { com questionário } \\
\text { autoaplicável }\end{array}$ & $\begin{array}{l}\text { Quatrocentos e nove alunos participaram; } 78 \% \text { eram } \\
\text { mulheres. O significativo a idade foi de } 21,6 \text { anos. Dor } \\
\text { no pescoço, cansaço visual, dor no ombro e olhos } \\
\text { ardendo foram os sintomas de SVC mais comuns. } \\
\text { Moderado visão turva foi relatada em dos que olharam } \\
\text { para o dispositivo em comparação com os que o } \\
\text { seguraram em ângulo adequado. }\end{array}$ & $\begin{array}{l}\text { Os sintomas oculares e dor muscular ocorriam } \\
\text { em menor número se o dispositivo fosse } \\
\text { segurado ao nível dos olhos. Há uma alta } \\
\text { prevalência de sintomas de SVC entre os } \\
\text { universitários que poderiam ser reduzidos, } \\
\text { como, dor no pescoço, cansaço visual e } \\
\text { queimação, com práticas ergonômicas } \\
\text { adequadas. }\end{array}$ \\
\hline SA EC, et al., 2012 & $\begin{array}{l}\text { Risk Factors for } \\
\text { Computer Visual } \\
\text { Syndrome (CVS) }\end{array}$ & $\begin{array}{l}\text { Estudo quantitativo } \\
\text { transversal } \\
\text { observacional e } \\
\text { análise ergonômica } \\
\text { do trabalho }\end{array}$ & $\begin{array}{l}\text { O horário de trabalho semanal das operadoras era de } 36 \\
\text { horas, com intervalo de } 21 \text { a } 35 \text { minutos por dia. Os } \\
\text { sintomas relatados foram fadiga ocular, "peso" nos } \\
\text { olhos, "ardor" nos olhos, lacrimejamento e } \\
\text { enfraquecimento da visão. A prevalência da SVC foi de } \\
54,6 \% \text {. }\end{array}$ & $\begin{array}{l}\text { Os resultados afirmaram a importância de } \\
\text { estudar e identificar a SVC entre operadoras } \\
\text { de call centers e que existe uma associação } \\
\text { entre o sintomas e condições de trabalho. }\end{array}$ \\
\hline
\end{tabular}




\begin{tabular}{|c|c|c|c|c|}
\hline $\begin{array}{l}\text { Autores e Ano de } \\
\text { Publicação }\end{array}$ & Titulo & Tipo de estudo & Resultados & Conclusão \\
\hline $\begin{array}{l}\text { RANASINGHE P, et } \\
\text { al., } 2016\end{array}$ & $\begin{array}{l}\text { Computer vision } \\
\text { syndrome among } \\
\text { computer office } \\
\text { workers in a } \\
\text { developing country: } \\
\text { an evaluation of } \\
\text { prevalence and risk } \\
\text { factors }\end{array}$ & $\begin{array}{l}2500 \text { trabalhadores } \\
\text { de escritórios de } \\
\text { informática do Sri } \\
\text { Lanka, entre maio e } \\
\text { dezembro de } 2009 \\
\text { participaram de um } \\
\text { estudo quantitativo } \\
\text { transversal. }\end{array}$ & $\begin{array}{l}\text { Sexo feminino com alta duração de ocupação, uso diário } \\
\text { de computador, doença ocular preexistente, uso de } \\
\text { lentes de contato e conhecimento de práticas } \\
\text { ergonômicas foram associados significativamente } \\
\text { presença de SVC. A duração da ocupação e a presença } \\
\text { de doença ocular preexistente foram associadas a } \\
\text { gravidade da doença. }\end{array}$ & $\begin{array}{l}\text { Os funcionários do Siri Lanka apresentaram } \\
\text { alta taxa de SVC, O Sexo feminino apresentou } \\
\text { maior duração, além disso, uso aumentado } \\
\text { diário de computador, doença ocular } \\
\text { preexistente, uso de lentes de contato e a falta } \\
\text { de conhecimento das práticas de ergonomia } \\
\text { todos foram associados com a presença de } \\
\text { SVC. }\end{array}$ \\
\hline $\begin{array}{c}\text { ROSENFIELD M, } \\
2011\end{array}$ & $\begin{array}{l}\text { Computer vision } \\
\text { syndrome: a review of } \\
\text { ocular causes and } \\
\text { potential treatments. }\end{array}$ & $\begin{array}{l}\text { Este artigo revisa a } \\
\text { SVC e as principais } \\
\text { causas para esta } \\
\text { condição e olho seco. }\end{array}$ & $\begin{array}{l}\text { A prevalência de sintomas de olho seco é maior durante } \\
\text { a operação do computador, ocorre provavelmente } \\
\text { devido a uma diminuição da taxa e amplitude de } \\
\text { piscadas, bem como aumento da exposição da córnea } \\
\text { resultante do monitor frequentemente ser posicionado } \\
\text { no olhar primário. }\end{array}$ & $\begin{array}{l}\text { Uma melhor compreensão da fisiologia } \\
\text { subjacente a SVC é fundamental para permitir } \\
\text { diagnóstico e tratamento mais precisos. Isso } \\
\text { permitirá que os profissionais otimizem o } \\
\text { conforto visual e a eficiência durante a } \\
\text { operação do computador }\end{array}$ \\
\hline $\begin{array}{c}\text { GOWRISANKARAN } \\
\text { S e SHEEDY JE, } \\
2015\end{array}$ & $\begin{array}{l}\text { Computer vision } \\
\text { syndrome }\end{array}$ & $\begin{array}{l}\text { Revisão de literatura } \\
\text { sobre SVC analisada } \\
\text { nos últimos } 65 \text { anos. }\end{array}$ & $\begin{array}{l}\text { Os sintomas relatados pelos usuários de computador } \\
\text { são divididos em oculares internos, oculares externos, } \\
\text { sintomas visuais e sintomas musculoesqueléticos. Os } \\
\text { principais fatores associados a SVC são ambientais } \\
\text { (iluminação inadequada, posição de exibição e distância } \\
\text { de visualização) e / ou dependente das habilidades } \\
\text { visuais do usuário (erro refrativo não corrigido, distúrbios } \\
\text { oculomotores) }\end{array}$ & $\begin{array}{l}\text { Embora os fatores associados a SVC tenham } \\
\text { sido identificados, os mecanismos fisiológicos } \\
\text { que fundamentam a SVC não são totalmente } \\
\text { compreendidos. Além disso, os avanços na } \\
\text { tecnologia levaram ao aumento do uso de } \\
\text { dispositivos portáteis, o que pode impor } \\
\text { desafios visuais um pouco diferentes em } \\
\text { comparação aos monitores de desktop. }\end{array}$ \\
\hline $\begin{array}{l}\text { ROSENFIELD M, } \\
2016\end{array}$ & $\begin{array}{l}\text { Computer vision } \\
\text { syndrome (a.k.a. } \\
\text { digital eye strain) }\end{array}$ & $\begin{array}{l}\text { Este artigo analisa as } \\
\text { principais causas } \\
\text { oculares para a SVC } \\
\text { e discute como o } \\
\text { exame oftalmológico } \\
\text { padrão deve ser } \\
\text { modificado para } \\
\text { atender às demandas } \\
\text { visuais da atualidade. }\end{array}$ & $\begin{array}{l}\text { Foi demonstrado que a fadiga ocular digital tem um } \\
\text { impacto significativo em ambos, conforto visual e } \\
\text { produtividade ocupacional, já que cerca de } 40 \% \text { dos } \\
\text { adultos e até } 80 \% \text { dos adolescentes podem } \\
\text { experimentar sintomas visuais significativos } \\
\text { (principalmente cansaço visual, olhos secos e } \\
\text { cansados), tanto durante quanto imediatamente após a } \\
\text { visualização eletrônica. }\end{array}$ & $\begin{array}{l}\text { Os profissionais da saúde ocular são } \\
\text { responsáveis pelo entendimento dos sintomas } \\
\text { associados e dos problemas fisiológicos } \\
\text { subjacentes durante a visualização de } \\
\text { monitores digitais. Como sociedade moderna } \\
\text { continua a avançar no sentido de um uso ainda } \\
\text { maior de dispositivos eletrônicos para } \\
\text { atividades de trabalho e lazer, apresentando } \\
\text { uma incapacidade de satisfazer os requisitos } \\
\text { visuais e dificuldades significativas no estilo de } \\
\text { vida dos paciente }\end{array}$ \\
\hline
\end{tabular}




\begin{tabular}{|c|c|c|c|c|}
\hline $\begin{array}{l}\text { Autores e Ano de } \\
\text { Publicação }\end{array}$ & Titulo & Tipo de estudo & Resultados & Conclusão \\
\hline $\begin{array}{c}\text { TAWIL LA, et al., } \\
2020\end{array}$ & $\begin{array}{l}\text { Prevalence of self- } \\
\text { reported computer } \\
\text { vision syndrome } \\
\text { symptoms and its } \\
\text { associated factors } \\
\text { among university } \\
\text { students }\end{array}$ & $\begin{array}{l}\text { Estudo transversal } \\
\text { com questionário } \\
\text { autoaplicável para } \\
713 \text { universitários da } \\
\text { Arábia Saudita }\end{array}$ & $\begin{array}{l}\text { O sintoma mais comum foi dor no pescoço ou ombro, } \\
\text { seguido por dor de cabeça e olhos secos, em leve, } \\
\text { moderada ou grave forma. O uso de dispositivos } \\
\text { eletrônicos por mais de } 5 \text { horas também foi associado a } \\
\text { sintomas de síndrome de visão computacional. }\end{array}$ & $\begin{array}{l}\text { A prevalência de sintomas da síndrome da } \\
\text { visão computacional foi significativamente } \\
\text { maior entre os estudantes de negócios, que } \\
\text { relataram menor consciência e medidas de } \\
\text { prática inadequada de recomendações de uso } \\
\text { do computador. Conscientização relevante, } \\
\text { campanhas com foco no uso adequado de } \\
\text { computadores são altamente recomendadas }\end{array}$ \\
\hline $\begin{array}{c}\text { PERIN AN, et al., } \\
2012\end{array}$ & $\begin{array}{l}\text { Computer-related } \\
\text { visual symptoms in } \\
\text { office workers }\end{array}$ & $\begin{array}{l}\text { Estudo transversal } \\
\text { com uso de } \\
\text { questionário } \\
\text { autoaplicável }\end{array}$ & $\begin{array}{l}\text { Os participantes que trabalharam com um ângulo de } \\
\text { visão inferior a } 10^{\circ} \text { em relação à tela apresentaram mais } \\
\text { sintomas, principalmente de dor na nuca e nas } \\
\text { costas. Trabalhadores mais jovens tiveram mais dores } \\
\text { de cabeça, e os trabalhadores com menos anos de } \\
\text { trabalho tiveram mais dores de cabeça e sintomas de } \\
\text { olhos secos. }\end{array}$ & $\begin{array}{l}\text { Participantes mais jovens com menos anos de } \\
\text { emprego, que não receberam informações } \\
\text { sobre o uso adequado do computador, quem } \\
\text { não utilizou iluminação entre } 450 \text { e } 699 \text { lux ou } \\
\text { quem trabalhou com ângulos de visão menor } \\
\text { do que dez graus apresentou mais sintomas da } \\
\text { SVC. }\end{array}$ \\
\hline $\begin{array}{c}\text { SANCHEZ V, et al., } \\
2020\end{array}$ & $\begin{array}{l}\text { Dry Eye Disease } \\
\text { Association with } \\
\text { Computer Exposure } \\
\text { Time Among Subjects } \\
\text { with Computer Vision } \\
\quad \text { Syndrome }\end{array}$ & $\begin{array}{l}\text { Realizado um estudo } \\
\text { transversal com } \\
\text { trabalhadores de } \\
\text { escritórios usuários } \\
\text { de computador }\end{array}$ & $\begin{array}{l}\text { O tempo médio de exposição ao computador foi } \\
\text { associado ao desenvolvimento do olho seco. Anos de } \\
\text { exposição ao computador e coloração de superfície } \\
\text { ocular apresentou correlação positiva significativa. O } \\
\text { acúmulo do tempo de exposição associado foi } \\
\text { negativamente correlacionado com a ruptura lacrimal e } \\
\text { positivamente com a integridade da superfície ocular. }\end{array}$ & $\begin{array}{l}\text { O tempo prolongado de exposição ao } \\
\text { computador em indivíduos com SVC foi } \\
\text { correlacionado significativamente com os } \\
\text { testes de olho seco, nas diferentes formas de } \\
\text { mensuração; mas não com o teste de } \\
\text { Schirmer. }\end{array}$ \\
\hline $\begin{array}{c}\text { AL RASHIDI SH e } \\
\text { ALHUMAIDAN H, } \\
2017\end{array}$ & $\begin{array}{l}\text { Computer vision } \\
\text { syndrome and Saudi } \\
\text { university students }\end{array}$ & $\begin{array}{l}\text { Estudo transversal } \\
\text { realizado em Qassim } \\
\text { university college } \\
\text { Medicina durante } \\
\text { período de } 1 \text { ano de } \\
\text { janeiro de } 2015 \text { a } \\
\text { janeiro de } 2016 \text {. }\end{array}$ & $\begin{array}{l}634 \text { alunos, foram incluídos no estudo com predomínio } \\
\text { do sexo masculino. Do total, a maioria apresentou } \\
\text { sintomas agudos, enquanto os restantes tinham } \\
\text { problemas crônicos. Uma clara maioria carregava os } \\
\text { sintomas por }<5 \text { dias e }>1 \text { mês. A análise estatística } \\
\text { revelou sintomas graves na maioria do estudo assuntos } \\
\text { especialmente aqueles que são usuários permanentes } \\
\text { de um computador por longas horas. }\end{array}$ & $\begin{array}{l}\text { O uso contínuo do computador por longas } \\
\text { horas e considerado grave, problemas de } \\
\text { visão, especialmente em quem usa } \\
\text { computadores e dispositivos semelhantes por } \\
\text { um longo período aparecem com grande } \\
\text { frequência. }\end{array}$ \\
\hline GUALOTO KQ, 2019 & $\begin{array}{l}\text { Factores de riesgo e } \\
\text { intervenciones } \\
\text { ergonômicas } \\
\text { efectivas para el } \\
\text { manejo del síndrome } \\
\text { de vision de } \\
\text { computadora. } \\
\end{array}$ & $\begin{array}{l}\text { Trata-se de uma } \\
\text { revisão bibliográfica }\end{array}$ & $\begin{array}{l}\text { As telas se tornaram ferramenta de trabalho } \\
\text { indispensável nas áreas técnicas administrativas, cujo } \\
\text { uso constante pode gerar um risco ergonômico em } \\
\text { usuários frequentes, conhecido como síndrome da visão } \\
\text { computacional. }\end{array}$ & $\begin{array}{l}\text { A análise das informações mostra que a } \\
\text { adequação da iluminação direta e mudanças } \\
\text { indiretas, ergonômicas dos equipamentos de } \\
\text { informática e correção de pequenas doenças } \\
\text { refratárias em que o uso de lentes não foi } \\
\text { considerado necessário, são controles } \\
\text { eficazes para este risco. }\end{array}$ \\
\hline
\end{tabular}




\begin{tabular}{|c|c|c|c|c|}
\hline $\begin{array}{l}\text { Autores e Ano de } \\
\text { Publicação }\end{array}$ & Titulo & Tipo de estudo & Resultados & Conclusão \\
\hline CRUZ C, et al., 2018 & $\begin{array}{l}\text { Síndroma Visual de } \\
\text { Computadores em } \\
\text { Trabalhadores de } \\
\text { Escritório }\end{array}$ & $\begin{array}{l}\text { Revisão bibliográfica } \\
\text { integrativa }\end{array}$ & $\begin{array}{l}\text { Os resultados encontrados demonstram, em todos os } \\
\text { estudos, uma elevada prevalência de SVC. Observam- } \\
\text { se diferenças entre estudos, relativamente aos sintomas } \\
\text { de Síndrome Visual de Computadores apresentados } \\
\text { pelos trabalhadores, podendo estes resultados estar } \\
\text { relacionados com as características de cada amostra, } \\
\text { assim como com os diferentes instrumentos utilizados } \\
\text { para avaliar as mesmas }\end{array}$ & $\begin{array}{l}\text { Os utilizadores de computador olham para um } \\
\text { monitor durante horas, levando a sintomas } \\
\text { como fadiga ocular e desconforto, causando, } \\
\text { na sua maioria, cefaleias, sintomatologia } \\
\text { caracterizada pela SVC. }\end{array}$ \\
\hline $\begin{array}{l}\text { NEVES LRC e FILHO } \\
\text { JJSD, } \\
2019\end{array}$ & $\begin{array}{l}\text { Estudo dos sintomas } \\
\text { visuais na síndrome } \\
\text { relacionada ao } \\
\text { computador e efeitos } \\
\text { dos colírios } \\
\text { lubrificantes em } \\
\text { funcionários do } \\
\text { hospital universitário } \\
\text { Bettina Ferro de } \\
\text { Sousa }\end{array}$ & $\begin{array}{l}\text { Estudo com } \\
\text { metodologia mista, } \\
\text { realizado no Hospital } \\
\text { Universitário Bettina } \\
\text { Ferro de Sousa } \\
\text { (HUBFS), Belém- } \\
\text { Pará, em funcionários } \\
\text { que utilizavam } \\
\text { computador }\end{array}$ & $\begin{array}{l}\text { A prevalência da síndrome foi de } 75,4 \% \text {. O sexo não } \\
\text { demonstrou significância estatística. Variáveis como } \\
\text { lluminação artificial inadequada, brilho excessivo da } \\
\text { tela, e número de horas/dia em frente ao computador, } \\
\text { apresentaram significância estatística na comparação } \\
\text { com a SVC. Sintomas como fadiga, cefaleia e } \\
\text { ressecamento, tiveram redução significativa após uso do } \\
\text { colírio. }\end{array}$ & $\begin{array}{l}\text { A SVC mostrou-se altamente prevalente na } \\
\text { população estudada. Foi comprovada } \\
\text { associação diretamente proporcional entre } \\
\text { número de horas/dia frente as telas e } \\
\text { quantidade de sintomas apresentados. O uso } \\
\text { de colírios lubrificantes reduziu os sintomas } \\
\text { relatados, assim como melhorou o } \\
\text { desempenho no trabalho. }\end{array}$ \\
\hline $\begin{array}{l}\text { GENTIL RM, et al., } \\
2011\end{array}$ & $\begin{array}{l}\text { Síndrome da visão do } \\
\text { computador }\end{array}$ & $\begin{array}{l}\text { Revisão integrativa } \\
\text { da literatura }\end{array}$ & $\begin{array}{l}\text { Longos períodos de trabalho sem pausas são } \\
\text { prejudiciais para os sintomas oculares. Intervalos } \\
\text { frequentes são recomendados para restaurar e relaxar o } \\
\text { sistema de acomodação e evitar a fadiga ocular. }\end{array}$ & $\begin{array}{l}\text { Importante compreender a SVC como uma } \\
\text { doença de abordagem multifatorial, } \\
\text { encontrando soluções eficientes e } \\
\text { impactantes, pois a saúde ocular é relevante } \\
\text { tanto para a segurança como para a } \\
\text { produtividade no trabalho. }\end{array}$ \\
\hline $\begin{array}{l}\text { REDDY SC, et al., } \\
2013\end{array}$ & $\begin{array}{l}\text { Computer vision } \\
\text { syndrome: a study of } \\
\text { knowledge and } \\
\text { practices in university } \\
\text { students }\end{array}$ & $\begin{array}{l}\text { Estudo transversal, } \\
\text { por questionário }\end{array}$ & $\begin{array}{l}795 \text { alunos, com idades entre } 18 \text { e } 25 \text { anos, de cinco } \\
\text { universidades da Malásia foram pesquisados. A } \\
\text { prevalência de sintomas de SVC (um ou mais) } \\
\text { encontrada foi de } 89,9 \% \text {; o sintoma mais preocupante } \\
\text { foi cefaleia ( } 19,7 \%) \text {, seguido de cansaço visual } \\
\text { (16,4\%). Os alunos que usaram o computador por mais } \\
\text { de } 2 \text { horas por dia apresentaram significativamente mais } \\
\text { sintomas de SVC. Olhar para objetos distantes entre o } \\
\text { trabalho foi significativamente associado a menos } \\
\text { frequência de sintomas SVC. }\end{array}$ & $\begin{array}{l}\text { Noventa por cento dos estudantes } \\
\text { universitários na Malásia experimentaram } \\
\text { sintomas relacionados ao CVS, que foi visto } \\
\text { com mais frequência em pessoas que usaram } \\
\text { o computador por mais de } 2 \text { horas } \\
\text { continuamente por dia. }\end{array}$ \\
\hline
\end{tabular}




\begin{tabular}{|c|c|c|c|c|}
\hline $\begin{array}{l}\text { Autores e Ano de } \\
\text { Publicação }\end{array}$ & Titulo & Tipo de estudo & Resultados & Conclusão \\
\hline $\begin{array}{l}\text { LEMMA MG, et al., } \\
2020\end{array}$ & $\begin{array}{l}\text { Computer Vision } \\
\text { Syndrome and } \\
\text { Associated Factors } \\
\text { Among Secretaries } \\
\text { Working in Ministry } \\
\text { Offices in Addis } \\
\text { Ababa, Ethiopia. }\end{array}$ & $\begin{array}{l}\text { Estudo transversal de } \\
\text { base institucional. }\end{array}$ & $\begin{array}{l}\text { A prevalência de SVC foi de } 68,8 \% \text {. Os principais } \\
\text { sintomas relatados foram visão turva }(36,9 \%) \text {, fadiga } \\
\text { ocular ( } 32,1 \%) \text {, vermelhidão do olho }(27,3 \%) \text { e cefaleia } \\
\text { (26,4\%). Renda média mensal, hábito de piscar } \\
\text { voluntariamente frequente, tomando intervalos } \\
\text { regulares entre o trabalho), usando óculos, fontes de luz } \\
\text { no local de trabalho, usando um filtro antirreflexo e } \\
\text { conhecimento foram significativamente associados com } \\
\text { CVS. }\end{array}$ & $\begin{array}{l}\text { A prevalência de CVS entre as secretárias foi } \\
\text { elevada. Os resultados sugerem a } \\
\text { necessidade de ajustar o tempo de exposição } \\
\text { aos computadores e aumentar } \\
\text { conscientização sobre medidas de segurança } \\
\text { e exames oftalmológicos regulares }\end{array}$ \\
\hline LURATI AR, 2018 & $\begin{array}{l}\text { Computer Vision } \\
\text { Syndrome } \\
\text { Implications for the } \\
\text { Occupational Health } \\
\text { Nurse }\end{array}$ & $\begin{array}{l}\text { Este artigo analisa a } \\
\text { patologia e as } \\
\text { intervenções para } \\
\text { SVC com implicações } \\
\text { para o enfermeiro. }\end{array}$ & $\begin{array}{l}\text { Computadores e dispositivos eletrônicos são usados no } \\
\text { local de trabalho e durante o lazer. A SVC é uma } \\
\text { condição que está afetando negativamente os } \\
\text { trabalhadores. }\end{array}$ & $\begin{array}{l}\text { Cada computador pode ser equipado com um } \\
\text { filtro antirreflexo para reduzir o cansaço visual } \\
\text { em reduzindo o brilho e melhorando as } \\
\text { imagens do computador, aumentando o } \\
\text { conforto ao operador. O brilho ou a luminância } \\
\text { deve ser ajustada para o ambiente. O uso de } \\
\text { óculos de bloqueio de comprimento de onda } \\
\text { curto pode diminuir a fadiga visual e o } \\
\text { desconforto }\end{array}$ \\
\hline $\begin{array}{c}\text { RANDOLPH AS, } \\
2017\end{array}$ & $\begin{array}{l}\text { Computer Vision } \\
\text { Syndrome }\end{array}$ & $\begin{array}{l}\text { Trata-se de uma } \\
\text { revisão de literatura }\end{array}$ & $\begin{array}{l}\text { Com aumento do uso de dispositivos eletrônicos, a } \\
\text { síndrome da visão do computador vem tornando-se um } \\
\text { grande problema de saúde pública. Melhorar o status } \\
\text { visual dos trabalhadores resulta em maior produtividade } \\
\text { no local de trabalho e melhor conforto. }\end{array}$ & $\begin{array}{l}\text { Após } 2 \text { horas de uso contínuo do computador, } \\
\text { os indivíduos devem descansar os olhos por } 15 \\
\text { minutos. Os funcionários também devem ser } \\
\text { encorajados a fazer exames oftalmológicos } \\
\text { regulares para manter a saúde visual e } \\
\text { prevenir SVC. }\end{array}$ \\
\hline
\end{tabular}

Fonte: Araújo IIO, et al., 2021. 
Longos períodos de trabalho sem pausas são prejudiciais para os sintomas oculares. Intervalos frequentes são recomendados para gerar relaxamento e restauração ao sistema de acomodação, evitando assim a fadiga ocular. Ao desviar o olhar para um objeto distante pelo menos duas vezes a cada hora durante o uso do computador seja suficiente para a prevenção da fadiga visual. Outros estudos compararam três intervalos diferentes $(60$ minutos de trabalho por 10 minutos de descanso, 30 minutos de trabalho por 5 minutos de descanso e 15 minutos de trabalho por microintervalos) em operadores de computador. Eles descobriram que o trabalho de 15 minutos e o cronograma de micro descanso produziram menos sintomas de dor no pescoço, na parte inferior das costas e no ombro; e que o esquema de 30 minutos de trabalho por 5 minutos de descanso produziu menos fadiga visual e visão turva (GENTIL RM, et al., 2011; REDDY SC, et al, 2013)

O uso de óculos de bloqueio de comprimento de onda curto pode diminuir a fadiga visual e o desconforto, contribuindo assim para a melhora da SVC. Segundo estudo, os vidros com filtro de luz azul não interferem no desempenho visual ou na qualidade do sono. Já as lentes coloridas são recomendadas, lentes cinza reduzem a luz de todo o espectro de luz. Enquanto as lentes de contato que bloqueiam os efeitos negativos da luz e a radiação pode fornecer mais proteção porque cobrem mais a superfície ocular do que óculos, contribuindo para uma diminuição da fadiga ocular e possivelmente a SVC (GOWRISANKARAN S e SHEEDY JE, 2015).

O design das condições de iluminação tem sido considerado um dos elementos mais importantes no design de locais de trabalho e está associado à produtividade e eficiência do trabalho. Numerosos estudos indicam que uma boa iluminação contribui para o desempenho no trabalho e para a redução das taxas de acidentes de trabalho (LEMMA MG, et al, 2020).

Em um estudo em que 18 escritórios de contabilidade participaram, sendo 113 funcionários participantes, constou-se que os participantes mais jovens com menos anos de emprego e que não receberam informações em relação ao uso adequado do computador, que não utilizou iluminação entre 450 e 699 lux ou quem trabalhou com ângulos de visão inferiores a $10^{\circ}$ apresentou mais sintomas da SVC (PERIN NA, et al., 2017). Com isso, pode-se reforçar a importância de informar sobre o uso do computador e a prevenção dos sintomas e da SVC, para a melhora da qualidade de vida da população.

Os funcionários de escritórios e usuários de equipamentos dotados de visor, têm como agravantes principais os fatores ambientais: a humidade do ar diminuída e a utilização de ar-condicionado, são os agentes desencadeadores de SVC em sua maioria de casos. A iluminação de forma errônea pode causar brilho no campo visual e contribuir para o desencadeamento de fadiga ocular; a posição do monitor afeta estes usuários. Um estudo indica o uso do filtro antirreflexo, já que este reduz o brilho e melhora o contraste do visor, gerando assim maior conforto visual para o usuário. Os níveis aceitáveis para uso do computador estão entre 300 e 500 lux. A luz natural é preferida para iluminar os locais de trabalho, embora por si só não garanta um contributo suficiente, pelo que deve ser compensada com luz artificial adequada de acordo com as necessidades e atividades a realizar (CRUZ C, et al., 2018; GUALOTO KQ, et al., 2019).

Vários estudos sugerem que a melhor abordagem à SVC deverá ser multidirecional, incluindo intervenções ao nível da ergonomia do posto de trabalho, da correção refrativa, da educação do utilizador, da iluminação e outros fatores ambientais, e do planeamento das pausas durante as tarefas de visualização ao computador. A ergonomia do posto de trabalho (cadeira, mesa, visor, teclado, rato e suporte de documentos) é um dos aspetos relevantes, uma vez que influencia as posturas adotadas, a distância e ângulo de visualização, e o brilho no monitor. No entanto, não é suficiente ter um posto de trabalho que permita um bom ajuste à população utilizadora, é necessário que os sujeitos estejam treinados para o ajustarem corretamente (GENTIL $\mathrm{RM}$, et al., 2011).

\section{CONSIDERAÇÕES FINAIS}

A SVC é uma série de distúrbios, que engloba o sistema ocular, músculo esquelético, bem como efeitos adversos psicológicos entre os usuários de telas. Para resumir, a transmissão de informações adequadas sobre o uso do computador, uso de monitores de maior frequência e resolução, filtros de tela, melhorando a 
facilidade de iluminação do ambiente, modificando o posicionamento ergonômico dos monitores; ajustes periféricos de computador como apoio para antebraço, teclado baixo e design de mouse vertical, pode beneficiar em lesões musculo esqueléticas recorrentes. O cuidado dos distúrbios da superfície ocular preexistente, trazem benefícios na manutenção da superfície ocular saudável e prevenção de sintomas relacionados. Portanto, sendo um resultado de múltiplas causas, exige uma intervenção sob medida para uma prevenção e tratamento eficazes, melhorando a qualidade de vida dessa população.

\section{REFERÊNCIAS}

1. AL RASHIDI SH, ALHUMAIDAN H. Computer vision syndrome and Saudi university students. International Journal of Health Sciences, 2017; 11(5).

2. CRUZ C, et al. Síndroma Visual de Computadores em Trabalhadores de Escritório. Revista Portuguesa de Saúde Ocupacional on line, 2018; 6: 1-12.

3. GENTIL RM et al. Síndrome da visão do computador. Science in Health, 2011; 2(1): 64-6.

4. GOWRISANKARAN S, SHEEDY JE. Computer vision syndrome. Work, 2015; 52: 303-314

5. GUALOTO KQ. Factores de riesgo e intervenciones ergonômicas efectivas para el manejo del síndrome de vision de computadora. Rev Ergon Invest Desar, 2019; 1(3): 10-21

6. LEMMA MG et al. Computer Vision Syndrome and Associated Factors Among Secretaries Working in Ministry Offices in Addis Ababa, Ethiopia. Clinical Optometry, 2020; 12: 213-222

7. LURATI AR. Computer Vision Syndrome Implications for the Occupational Health Nurse. Workplace Health \& Safety, February 2018; 66(2).

8. MOWATT $L$ et al. Computer vision syndrome and ergonomic practices among undergraduate university students. John Wiley \& Sons Ltd, 2017.

9. MUNSHI S, et al. Computer vision syndrome-A common cause of unexplained visual symptoms in the modern era. John Wiley \& Sons Ltd, 2017.

10. NEVES LRC, FILHO JJSD. Estudo dos sintomas visuais na síndrome relacionada ao computador e efeitos dos colírios lubrificantes em funcionários do hospital universitário Bettina Ferro de Sousa. Reas/Ejch, 2019; 11(8): e591.

11. PARILHAR JKS, et al. Computer \& visual display terminals (VDT) vision syndrome (CVDTS). Med J Armed Forces India, 2016.

12. PERIN AN, et al. Ergophthalmology in accounting offices: the computer vision syndrome (CVS). Rev Bras Oftalmol, 2017; 76(3): 144-9.

13. PORTELLO JK, et al. Computer-related visual symptoms in office workers. Ophthalmic Physiol Opt, 2012; 32: 375382.

14. RANASINGHE $P$, et al. Computer vision syndrome among computer office workers in a developing country: an evaluation of prevalence and risk factors. BMC Res Notes, 2016; 9: 150

15. RANDOLPH AS. Computer Vision Syndrome. Workplace Health \& Safety, 2017.

16. REDDY SC, et al. Computer vision syndrome: a study of knowledge and practices in university students. Nepal $J$ Ophthalmol, 2013; 5(2): 161-8.

17. ROSENFIELD M. Computer vision syndrome (a.k.a. digital eye strain). Optometry in Practice, $2016 ; 17$.

18. ROSENFIELD M. Computer vision syndrome: a review of ocular causes and potential treatments. Ophthalmic Physiol Opt, 2011; 31: 502-515.

19. SÁ EC, et al. Risk Factors for Computer Visual Syndrome (CVS). Work, 2012; 41: 3568-3574.

20. SANCHEZ V, et al. Dry Eye Disease Association with Computer Exposure Time Among Subjects with Computer Vision Syndrome. Clinical Ophthalmology, 2020; 14: 4311-4317.

21. TAWIL LA, et al. Prevalence of self-reported computer vision syndrome symptoms and its associated factors among university students. European Journal of Ophthalmology, 2020; 30(1): 189- 195. 